\title{
A Hybrid Illumination Refinement Technique Based on Double Niblack Thresholds
}

\author{
Yi-Hua Wang, Jung-San Lee, and Bo Li
}

\begin{abstract}
Image archive is a transformation from source to its digital form. This process, however, often leads to the irregular distribution of image illumination, which distorting the content of images. In this article, we propose a hybrid illumination technique based on double Niblack thresholds to refine the brightness of under-exposure and over-exposure images, including the text image and the text-photo image. Experimental results have shown that the new method can effectively adjust the illumination to improve the quality of digital images.
\end{abstract}

Index Terms-Illumination, contrast enhancement, image binarization, Niblack.

\section{INTRODUCTION}

In recent years, communication technologies have greatly advanced and brought the world into a brand new era of digital storage. Nowadays, people use photographing and scanning to record their lives. That is to transform the memory episode into digital form. Poor contrast and exposure issues, however, happen a lot in photographing or scanning which are shown in Fig. 1 and Fig. 2. To deal with these problems, image binarization and contrast enhancement are mostly used, which are working for different kinds of images. The image binarization technique has been applied on text images, while the contrast enhancement one has been used to process photo images.

Image binarization methods can be classified into two types: Global thresholding and Local thresholding. The most classical method in the first area is Otsu [1], and the Niblack [2] is the most representative one in the second area. Otsu is a global thresholding method processing the whole image with one single threshold. On the contrary, Niblack is a local thresholding method that measures the local statistics within a window for each pixel and adjusts the threshold according to local statistics information.

Contrast enhancement methods can also be classified into two categories: direct enhancement and indirect enhancement. Direct enhancement methods generally improve the image contrast by modifying the pixel value according to the image feature, such as edge detection. But this kind of method cannot deal with images that contain both text and photo. Indirect enhancement methods usually process the image by redistributing the probability density. The most outstanding

Manuscript received April 4, 2015; revised November 5, 2015. This work was supported by MOST103-2221-E-035-055-

Y. H. Wang and J. S. Lee are with Feng Chia University, Taichung, Taiwan (e-mail: leejs@fcu.edu.tw).

B. $\mathrm{Li}$ is with the Department of Electrical Engineering and Computer Science, Vanderbilt University, Tennessee, USA.

indirect enhancement technique is the histogram equalization method, and it can be further divided into two types, global histogram equalization and local histogram equalization [3], [4]. In the global histogram equalization technique, the whole image information is used as the input of its transformation function. This kind of method is suitable for the whole image contrast enhancement. In particular, it can perform perfectly with the image which has a histogram concentrated in a limited range. But it cannot deal with the local feature enhancement. The feature might be lost after the enhancement. It is due to the fact that the result of general global histogram equalization often diverges on high frequency region and converges on the low frequency region. As to the local histogram equalization technique, it applies a sliding window to obtain the local histogram and uses the local information to hold the contrast enhancement. In this case, it can greatly enhance the contrast of local features. Nevertheless, it might cause over-enhancement situations because it only enhances the image with local information.

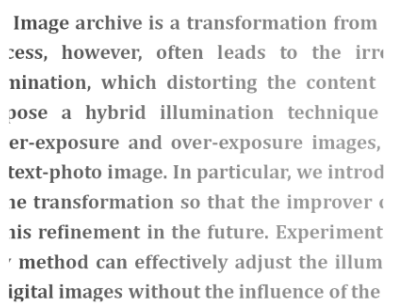

Image archive is a transformation from zess, however, often leads to the irr nination, which distorting the content ose a hybrid illumination technique er-exposure and over-exposure images, text-photo image. In particular, we intro e transformation so that the improver is refinement in the future. Experiment method can effectively adjust the illun igital images without the influence of the

(a)

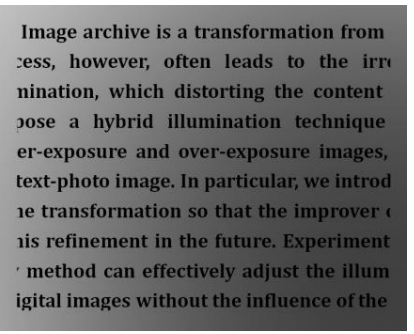

(b)

Fig. 1. Text image (a) Over-exposure, (b) Under-exposure

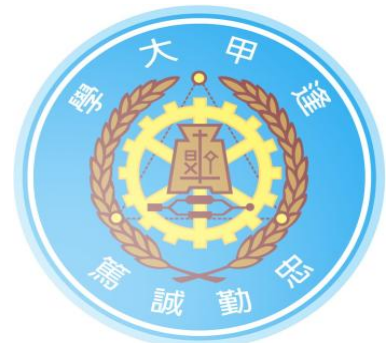

(a)

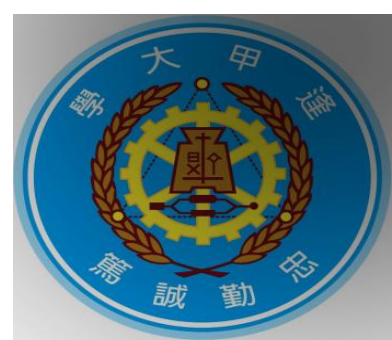

(b)
Fig. 2. Photo image (a) Over-exposure, (b) Under-exposure.

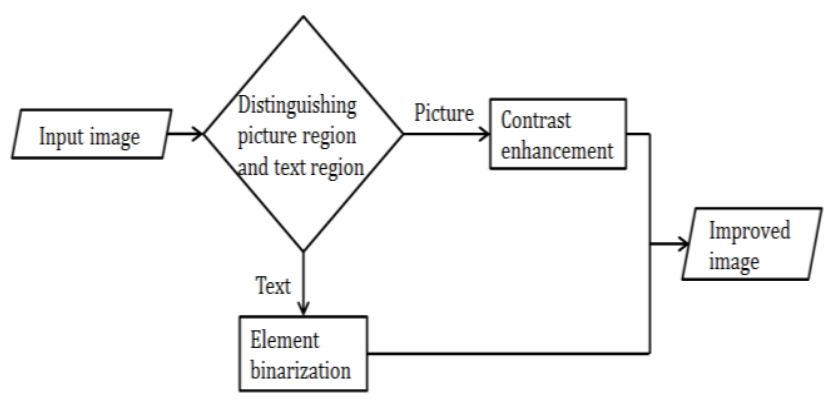

Fig. 3. Flowchart of the proposed scheme. 


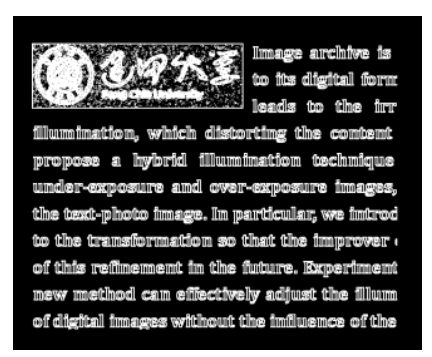

(a)

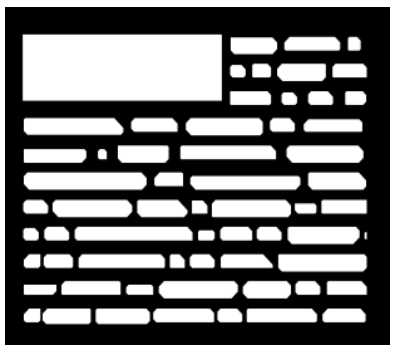

(b)

Fig. 4. Illustration of (a) Sobel detection and (b) marked edge regions.

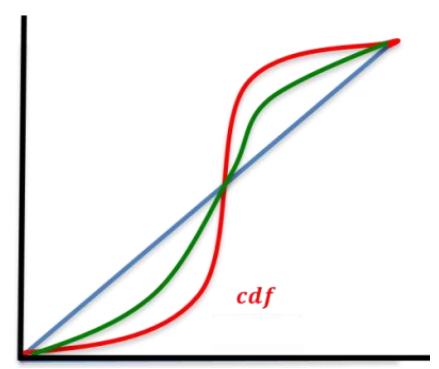

Fig. 5. Improvement curves.

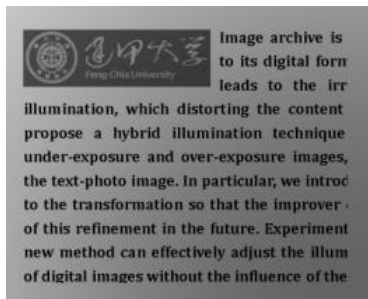

(a)

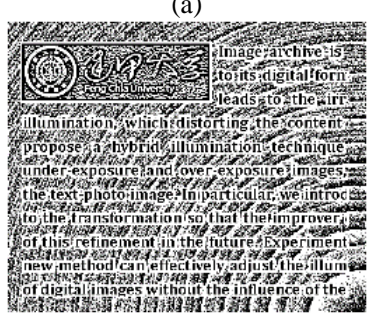

(c)

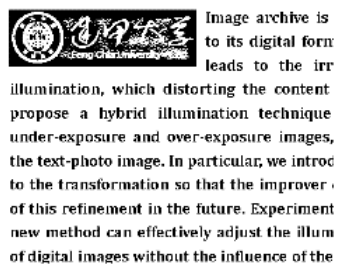

(b)

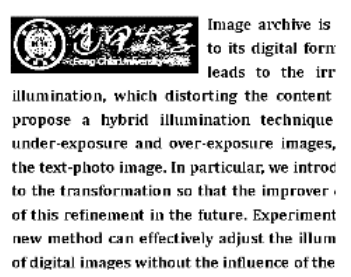

(d)
Fig. 6. (a) Original input image, Nibalck results with window sized (b) $15 \times$ 15 , (c) $30 \times 30$, (d) foreground image.

To process document image that contains text contents, photos or both of them, there are several methods have been proposed. This kind of methods can be classified into three categories by their achievements: 1) work on text-only images, 2) process text image or photo image, and 3) deal with text-photo image.

Hsia and Tsai have proposed a light-balancing technique (ELBT) that focuses on processing text images [5]. After separating the text part and the background area, the authors apply the linear interpolation to obtain new values that are suitable for the text pixels. Thus, a light balanced text image could be achieved by an adaptive gain control technique. In 2012, Chang et al. have developed an edge-based light balancing scheme (ELBS) [6]. The authors indicate the text part by the edge map that is generated by the Sobel technique, and they further locate the text pixels by merging the contrast enhanced and edge detected results. After that, they replace the located text pixels with new values to obtain the light distribution image and construct the light balanced image by merging the contrast enhanced and light distribution images. However, ELBT and ELBS can only adjust the illumination of text-only images.
In 2009, Lee et al. have designed an illumination balance technique (SIBT) for both text-only and text-photo images [7]. First, SIBT applies Sobel technique to determine the text and photo objects. Then it adjusts the illumination according to the light distribution of the whole image. Although SIBT can adjust the illumination of objects inside the under-exposure images, it is still invalid for images with over-exposure.

To process text image and text-photo image which may be under-exposure or over-exposure, we propose a hybrid image processing method which combines contrast enhancement and image binarization. The simulation results have demonstrated that the new method can achieve satisfactory performance.

The rest of this paper is organized as follows. In Section II, we introduce the details of the proposed scheme, followed by the experimental results and comparisons with the previous works in Section III. Finally, we make the conclusions.

\section{THE Proposed SCHEME}

The flowchart of the proposed method is shown in Fig. 3. The first step is to apply the edge detection technique to separate the pictures and texts, as described in subsection 2.1. Then we introduce how to perform the contrast enhancement on picture regions and how to execute binarization on text regions and image background in subsections 2.2 and 2.3, respectively. After combining all these intermediate results, we can obtain an improved image.

\section{A. Distinguishing Picture Regions and Text Regions}

To ensure that the improved image holds the same feature information instead of the brightness, we transform a RGB image into a HSV (Hue, Saturation, and Value) image. By only modifying the Value (Brightness) of the image, we can retain Hue and Saturation. In other words, we apply the Value information to obtain a gray level version of input image. Then we employ the Sobel detection technique [8] to contour and modify the edges into white color, and paint the remainder into black, as shown in Fig. 4(a). After that, we stretch the edges by tripling the width to avoid a broken situation. Next we mark the edge regions by checking the adjacent edge pixels. If there exist more than three white points in the $3 \times 3$ window which centers on point $p$, we modify the point $p$ into a white one. We carry on this procedure until there is no more black point. The result is shown in Fig. 4(b). We subsequently use Eq. (1) to determine whether the region is a text region or a picture region.

$$
\text { if }\left\{\begin{array}{lr}
\frac{\text { reigon area }}{\text { image size }}>0.02 \text { and } \frac{\text { region } \text { width }}{\text { region height }}<15, & \text { picture region } \\
\text { otherwise, } & \text { text region }
\end{array}\right.
$$

\section{B. Contrast Enhancement on Picture Regions}

To apply the contrast enhancement on picture regions, we first gather the statistics of pixel values. Then we can obtain the maximum value and the minimum value which the quantity should be greater than 30 . Next, we normalize the pixel values from $\max _{\text {new }}$ to $\min _{\text {new }}$, where $\max _{\text {new }}=$ the maximum value +40 , and $\min _{\text {new }}=$ the minimum value -40 . 
In this way, we can enlarge the range of the pixel value to enhance the processing extent of this procedure. After that, we measure the probability density function $(p d f)$ which can be calculated by Eq. (2) and the cumulative distribution function $(c d f)$ that is given in Eq. (3) of this region.

$$
\begin{gathered}
p d f(i)=\frac{n_{i}}{n} \\
c d f(i)=\sum_{j=0}^{i} p d f(i)
\end{gathered}
$$

where $n_{i}$ is the quantity of pixels that hold value $i$ in this region,

\begin{abstract}
Image archive is a transformation from ress, however, often leads to the irm nination, which distorting the content sose a hybrid illumination technique er-exposure and over-exposure images, text-photo image. In particular, we introd re transformation so that the improver lis refinement in the future. Experiment method can effectively adjust the illum igital images without the influence of the
\end{abstract}

(a)

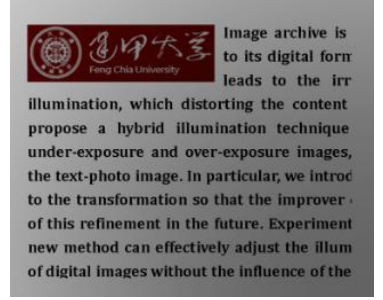

(e)

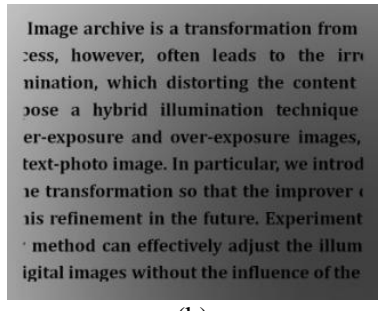

(b)

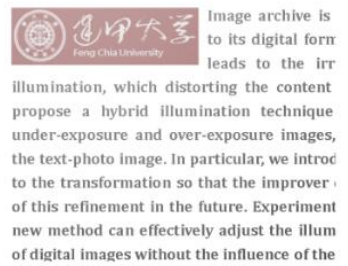

(f) and $n$ is the total number of pixels in this region.

After obtaining $c d f$ curve, we force the improvement curve imp (green curve in Fig. 5) approaching to the uniform distribution curve (blue curve in Fig. 5) and measure imp with Eq. (4). Then we modify every region according to its imp curve.

$$
\operatorname{imp}(i)=\frac{u n i f(i)-c d f(i)}{2}
$$

where unif is the uniform distribution curve.

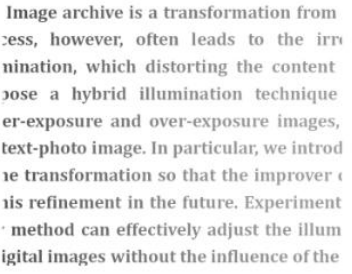

(c)

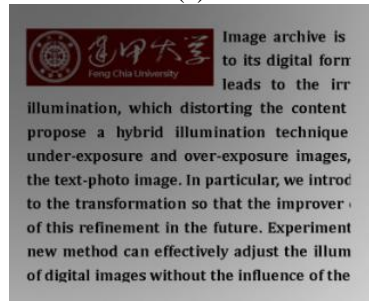

(g)

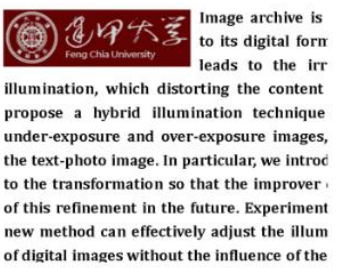

(d)

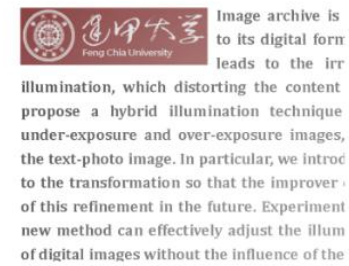

(h)

\begin{tabular}{|c|c|c|c|c|c|}
\hline \multicolumn{3}{|c|}{ Under-exposure } & \multicolumn{3}{|c|}{ Over-exposure } \\
\hline $\begin{array}{l}\text { Image archive is a transformation from } \\
\text { eess, however, often leads to the irrn } \\
\text { nination, which distorting the content } \\
\text { cose a hybrid illumination technique } \\
\text { er-exposure and over-exposure images, } \\
\text { text-photo image. In particular, we introd } \\
\text { re transtormation so that the improver, } \\
\text { lis refinement in the fature. Experiment } \\
\text { method can effectively ad|ust the illum } \\
\text { igital images without the influence of the }\end{array}$ & $\rightarrow$ & 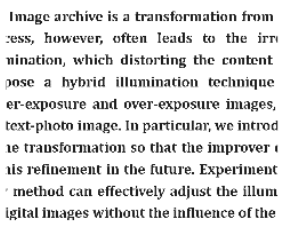 & $\begin{array}{l}\text { Image archive is a transformation from } \\
\text { :ess, however, often leads to the irre } \\
\text { nination, which distorting the content } \\
\text { jose a hybrid illumination technique } \\
\text { er-exposure and over-exposure images, } \\
\text { text-photo image. In particular, we introd } \\
\text { e transformation so that the improver , } \\
\text { is refinement in the future, Experiment } \\
\text { method can effectively adjust the illum } \\
\text { igital images without the influence of the }\end{array}$ & $\rightarrow$ & $\begin{array}{l}\text { Image archive is a transformation from } \\
\text { cess, however, often leads to the irr } \\
\text { nination, which distorting the content } \\
\text { pose a hybrid illumination technique } \\
\text { er-exposure and over-exposure images, } \\
\text { text-photo image. In particular, we introd } \\
\text { e transformation so that the improver ' } \\
\text { nis refinement in the future. Experiment } \\
\text { method can effectively adjust the illum } \\
\text { igital images without the influence of the }\end{array}$ \\
\hline 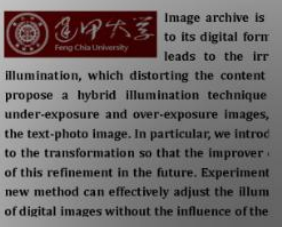 & $\rightarrow$ & 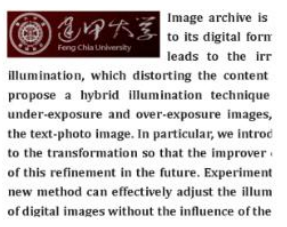 & 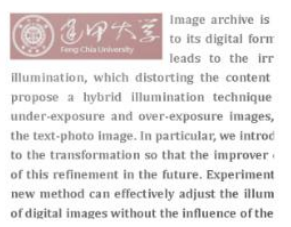 & $\rightarrow$ & 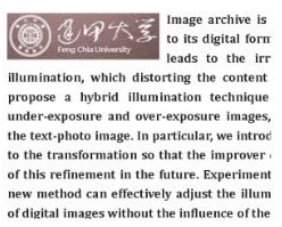 \\
\hline 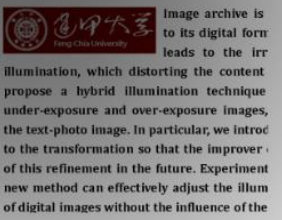 & $\rightarrow$ & $\begin{array}{l}\text { Image archive is } \\
\text { to its digital form } \\
\text { leads to the irr } \\
\text { illumination, which distorting the content } \\
\text { propose a liybrid illumination techinique } \\
\text { under-exposure and over-exposure images, } \\
\text { the text-photo image. In particular, we introc } \\
\text { to the trastorormation so that the improver. } \\
\text { of this refinement in the future. Experiment } \\
\text { new method can effectively adjust the illum } \\
\text { of digital images without the influence of the }\end{array}$ & 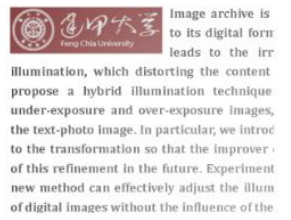 & $\rightarrow$ & 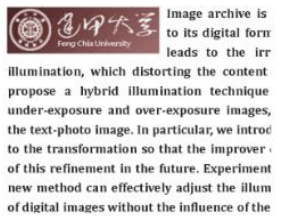 \\
\hline
\end{tabular}

Fig. 7. (a) and (d) are original images, (b)-(c) and (e)-(h) are imbalanced images.

TABLE I: EXPERIMENTAL RESULTS ON UNDER-EXPOSURE AND OVER EXPOSURE IMAGES

\section{Binarization on Text Regions and Image Background}

Here we introduce how to apply double Niblack algorithm to depart foreground (including texts and pictures) from background. We compute the first threshold for each pixel with a window $15 \times 15$ and determine the pixel value by Eq. (5); 255 and 0 are seen to be the background and the foreground. Next, we measure the threshold $T$ which is given in Eq. (6). The illustration result is shown in Fig. 6(b). Again, we calculate the second threshold for each pixel with a window $30 \times 30$. Then we can determine the pixel value and the threshold by Eq. (5) and Eq. (6), respectively. The result is display as Fig. 6(c)

$$
\begin{gathered}
\text { if }\left\{\begin{array}{lr}
\text { pixel value }>T, & 255 \\
\text { otherwise, } & 0
\end{array}\right. \\
T=\mu+k \times \sigma
\end{gathered}
$$


where $\mu$ is the standard deviation of this window, $k$ is the adjust parameter set to be $(-0.2)$, and $\sigma$ is the mean value of this window.

Once we adopt a smaller window in performing the Niblack, we may obtain a rigorous result keeping the contour of the foreground information, as shown in Fig. 6(b). On the contrary, we can obtain a rough image containing all of the foreground information with a larger window, as shown in Fig. 6(c). We subsequently overlap the contour image on the rough image, and mark all adjacent foreground pixels connected to the contour pixel. Thus we can obtain the refined text regions, as shown in Fig. 6(d).

Finally, we combine the picture region of subsection 2.2 and the text region of the subsection 2.3 to complete an improved image.

TABLE II: EXPERIMENTAL RESULTS ON UNDER-EXPOSURE IMAGES

\begin{tabular}{l} 
Text-only image \\
Image archive is a transformation from \\
zess, however, often leads to the irr \\
nination, which distorting the content \\
sose a hybrid illumination technique \\
er-exposure and over-exposure images, \\
text-photo image. In particular, we introd \\
1e transformation so that the improver c \\
1is refinement in the future. Experiment \\
method can effectively adjust the illum \\
igital images without the influence of the \\
\hline Test image \\
Image archive is a transformation from \\
zess, however, often leads to the irr \\
nination, which distorting the content \\
jose a hybrid illumination technique \\
er-exposure and over-exposure images, \\
text-photo image. In particular, we introd \\
1e transformation so that the improver c \\
1is refinement in the future. Experiment \\
method can effectively adjust the illum \\
igital images without the influence of the
\end{tabular}

\section{EXPERIMENTAL RESULTS AND COMPARISONS}

In this section, we illustrate the experimental results with nine different images that are shown in Fig. 7. Fig. 7(a) is an original image without picture region, sized $856 \times 684$ pixels, while Fig. 7(d) is a perfect image containing both picture and text regions, sized $696 \times 566$ pixels. Fig. 7(b) and Fig. 7(c) illustrate the under-exposure and over-exposure versions of Fig. 7(a), in which the light sources are from the top-left and bottom-right, respectively. Figs. 7(e) and 7(f) display the same mixed image of Fig. 7(d), in which the light source is from top-left, where Fig. 7(e) is an under-exposure image and Fig. 7(f) is an over-exposure one. Similarly, Figs. 7(g) and 7(h)
Text-photo image

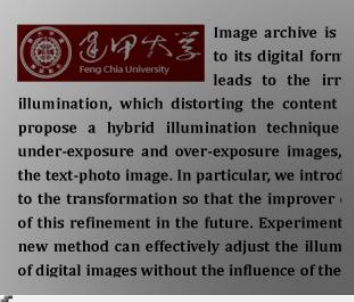

Image archive is to its digital form illumination, which distorting the content propose a hybrid illumination technique under-exposure and over-exposure images, the text-photo image. In particular, we introo to the transformation so that the improver orthis refrement in the fut net in can enectively adjust the illum of digital images without the influence of the

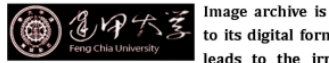
illumination, which distorting the content propose a hybrid illumination technique under-exposure and over-exposure images, the text-photo image. In particular, we introd to the transformation so that the improver, of this refinement in the future. Experiment of this refinement in the future. Experiment of igita

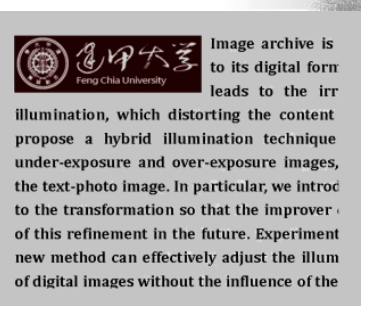

(9) leads to the irr illumination, which distorting the content propose a hybrid illumination technique under-exposure and over-exposure images, the text-photo image. In particular, we introc to the transformation so that the improver of this refinement in the future. Experiment new method can effectively adjust the illum of digital images without the influence of the
Text-photo image
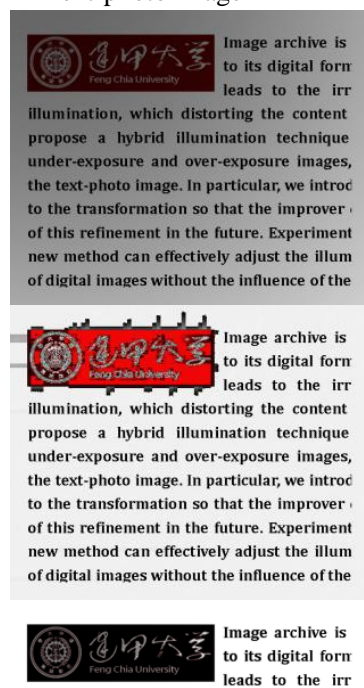
leads to the irr illumination, which distorting the content propose a hybrid illumination technique under-exposure and over-exposure images the text-photo image. In particular, we introc to the transformation so that the improver of this refinement in the future. new method can effectively adjust the illum digital imases without the influ

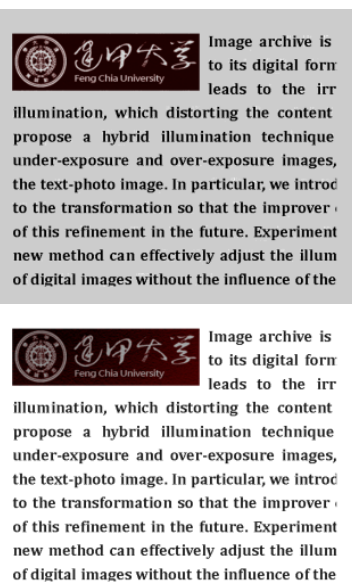

depict the under-exposure and over-exposure mixed images with the light source from bottom-right.

Table I displays the results of the proposed method on these under-exposure and over-exposure images. It is obvious that the new method can effectively improve the imbalanced illumination. In particular, the text and background regions of the improved images are extremely close to those of the original images. It is due to fact that we have distinguished the text region and picture region and applied the image binarization technique on the text and background region.

To demonstrate the superiority of the proposed scheme, we also simulated related works and made the comparisons in Table I and Table II. In Table II, we examined the ability for 
renovating the under-exposure images, and it contains text-only image and text-photo images with different directions of light source. Though ELBT and ELBS can refine the text-only image, they cannot deal with the text-photo images. Concerned to the SIBT, it seems that both the text-only and text-photo images could be renovated. Nevertheless, a serious under-exposure phenomenon will lead to a dark background of the improved image. This has lowered down the quality of the digital image. As to the proposed method, all the text region, photo region, and background can be renovated well.

On the other hand, we have explored the capability of related works on refining the over-exposure images. The corresponding results have been shown in Table II. Unfortunately, ELBT and ELBS cannot improve the imbalanced background. For SIBT, since it only measures the brightness of the background with the imbalanced pixel value, it can obtain a balance background instead of recovering the true color of the original image. No doubt that the proposed method can achieve a better performance that related works.

\section{CONCLUSIONS}

In this article, we have proposed a hybrid illumination refinement technique containing four phases. In the first phase, we distinguish the image into picture regions and text regions. Next, we apply the contrast enhancement to improve the picture regions. Third, we adopt double Niblack to renovate the text regions. Then, we combine intermediate results to obtain the final result. The experimental results and comparisons have demonstrated the contribution of the proposed method.

\section{REFERENCES}

[1] N. Otsu, "A threshold selection method from gray-level histograms," IEEE Transactions on Systems Man and Cybernetics, vol. 20, pp. 62-66, 1979.

[2] W. Niblack, An Introduction to Digital Image Processing, Prentice-Hall, pp. 115-116, 1986.
[3] S. C. Huang, F. C. Cheng, and Y. S. Chiu, "Efficient contrast enhancement using adaptive gamma correction with weighting distribution," IEEE Transactions on Image Processing, vol. 22, issue 3, pp. 1032-1041, March 2013.

[4] Z. Zhou, N. Sang, and X. Hu, "Global brightness and local contrast adaptive enhancement for low illumination color image," Optik International Journal for Light and Electron Optics, vol. 125, issue 6, pp. 1795-1799, March 2014

[5] S. C. Hsia and P. S. Tsai, "Efficient light balancing techniques for text images in video presentation systems," IEEE Transactions on Circuits and Systems for Video Technology, vol. 15, issue 8, pp. 1026-1031, 2005.

[6] K. N. Chen, C. H. Chen, and C. C. Chang, "Efficient illumination compensation techniques for text images," Digital Signal Processing, vol. 22, issue 5, pp. 726-733, September 2012.

[7] J. S. Lee, C. H. Chen, and C. C. Chang, "A novel illumination-balance technique for improving the quality of degraded text-photo images," IEEE Transactions on Circuits and Systems for Video Technology, pp. 900-905, 2009.

[8] I. Sobel, History and Definition of the Sobel Operator, 2014.

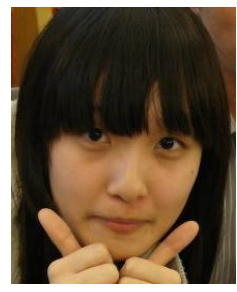

Yi-Hua Wang is currently pursuing her MS degree in information engineering and computer science in Feng Chia University, Taichung, Taiwan. Her current research interests include image processing and watermarking.

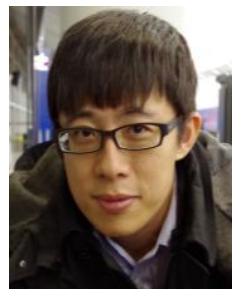

Jung-San Lee has worked as an associate professor in the Department of Information Engineering and Computer Science at Feng Chia University, Taichung, Taiwan. His current research interests include information security, image processing, and watermarking.

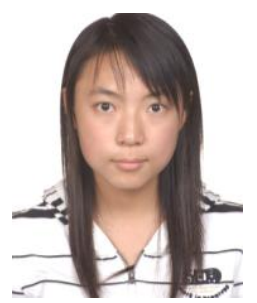

Bo Li is currently pursuing her Ph.D. degree in electrical engineering and computer science in Vanderbilt University, Tennessee, USA. Her current research interests include secret sharing technique and mobile communications. 